\title{
Mixed Agro-waste Biocomposites of Low Density Polyethene; Impact of Fillers on Mechanical, Morphological, Water Imbibition and Biodegradability Properties
}

\author{
Marcellinus Chibuike Ogudo", Pauline Uchechukwu Chris-Okafor, Joy Nwando Nwokoye, \\ Juliana Ozioma Anekwe
}

Department of Pure and Industrial Chemistry, Nnamdi Azikiwe University, Awka, Nigeria

Email address:

ogudomarcellinus@yahoo.com (M. C. Ogudo), pu.chrisokafor@unizik.edu.ng (P. U. Chris-Okafor)

${ }^{*}$ Corresponding author

To cite this article:

Marcellinus Chibuike Ogudo, Pauline Uchechukwu Chris-Okafor, Joy Nwando Nwokoye, Juliana Ozioma Anekwe. Mixed Agro-waste Biocomposites of Low Density Polyethene; Impact of Fillers on Mechanical, Morphological, Water Imbibition and Biodegradability Properties. American Journal of Polymer Science and Technology. Vol. 7, No. 3, 2021, pp. 44-49. doi: 10.11648/j.ajpst.20210703.12

Received: August 1, 2021; Accepted: August 12, 2021; Published: August 23, 2021

\begin{abstract}
In retrospect, the incorporation of agricultural waste as a natural, biodegradable filler in polymer composites is a scientific innovation that has received global attention. This is due to its impact in enhancing sustainability of composites and degradation of plastics. The mechanical, morphological, water imbibition and biodegradability properties of low density polyethene produced composites were investigated using agro wastes blend of corn cobs and sugarcane bagasse. Agro waste with a particle size of $75 \mu \mathrm{m}$ was blended in a 50:50 ratio and integrated into LDPE polymer matrix at different percentages of $0 \%, 5 \%, 10 \%, 15 \%$, and $20 \%$, with composites fabricated via injection molding. The mechanical properties like the tensile strength, percentage elongation at break, compressive strength, shear modulus and hardness test of the composites were studied in line with ASTM standards. Also, the morphological properties were studied with a Scanning Electron Microscope. From the results, there was a reduction in tensile strength of the composites with increasing filler loading. However, the percentage elongation at break of the LDPE composite increased as the filler loading increased as an indication that the composite is relatively ductile. Hardness, compressive strength and shear modulus of the polymer composites increased with increasing filler loading. Morphological study of the composites showed a good adhesion and interfacial bonding between the filler and the polymer matrix due to good dispersion of the fillers in the polymer matrix. Biodegradation test revealed a reduction in the mass of the composites after a 3- month burial period which was more pronounced after a 30-day test period indicating that the composite is more environmentally friendly. The highest degradation rate was observed at 20wt\% CCSB-LDPE composite giving a $15 \%$ reduction after 90 days. Result from the water imbibition test indicated no increase in the mass of the composites after immersion in water. This is an indication that the composite can be utilized in wet environments. Hence, from the findings, it is suggested that agro-wastes like rice husk and sugarcane bagasse be employed as fillers in the manufacture of plastics because they are cheaper and viable. Finally, the produced plastics would degrade when discarded.
\end{abstract}

Keywords: Agro-waste, Low Density Polyethene, Mechanical and Morphological Properties, Water Absorption, Biodegradation

\section{Introduction}

Increased activity in the modern agriculture industry generates a significant amount of rubbish, posing a substantial environmental concern. Plastics' relevance and wide range of applications in man's daily existence cannot be overstated.

Meanwhile, raw resource scarcity is causing concern, and agricultural waste could be a viable alternative for generating value-added goods such as bio-composites in this context. As a long-term strategy for exploiting the tremendous amount of natural plant fiber that is now underutilized, natural fibers, particularly agricultural waste fibers, require further development.

Lignocellulose fillers are a scientific and technological innovation in the field of novel materials, emphasizing the 
use of agricultural waste as a source of raw materials. As a result of their high specific strength, high modulus, reusability, green origin, large scale application, easy workability, and relatively cheap, lignocellulosic composites have gained a lot of interest.

Eco friendly composites have made significant progress since they are non-toxic, biodegradable, and safer to work with $[1,2]$.

Incorporating plant fibers/fillers such as sugar cane, soy protein, rice husk, coconut husk and cotton stalk as a natural filler is important for reinforcing polymers [3-9].

The usage, reuse, and recycling of agricultural waste, as well as the addition of natural filler to the composite material, can help to mitigate environmental issues related with their accumulation $[10,11]$. With the exception of fibrous natural fillers, the loading of dispersed agricultural waste in the polymer matrix is currently receiving a lot of attention. As a result, lignocellulosic plant waste, such as shells, husks, and stems of agricultural crops, can be used not only as a source of biomass, but also as low-cost dispersion fillers for polymer composites.

The aim of this study is to investigate the suitability of corn cobs and sugarcane bagasse as fillers for the production of Low Density Polyethene composites.

\section{Materials and Methods}

\subsection{Materials}

LDPE (NGL015FS) produced by Indorama Eleme Petrochemicals, Nigeria, were employed in this study. Corn cobs and sugarcane baggase were obtained from Ajegunle, Lagos State, Nigeria. The agricultural waste materials were washed and sun dried for three days before being crushed and sieved locally and repeatedly using a grain mill machine M6FFC-270 to a fine powder of $75 \mu \mathrm{m}$ size.

\subsection{Preparation of Composites}

The virgin crystalline pellets of LDPE were weighed at $190 \mathrm{~g}, 180 \mathrm{~g}, 170 \mathrm{~g}$, and $160 \mathrm{~g}$ and thoroughly mixed with a 50:50 blend of corn cobs and sugarcane bagasse fillers to yield LDPE composites. The weight of the blend varied from $10 \mathrm{~g}, 20 \mathrm{~g}, 30 \mathrm{~g}$, and $40 \mathrm{~g}$, corresponding to $5 \%, 10 \%, 15 \%$, and $20 \%$ filler loading, respectively. The homogeneous polymer and filler mixture was put into the hopper of a TU150 200 gram injection molding machine with a circular shaped mold. For mechanical property analysis, the composites were formed into standard dimensions.

\subsection{Mechanical Properties Analysis of Composites}

\subsubsection{Tensile Strength}

This is the composites' ability to endure loads that tend to elongate - in other words, the greatest amount of stress that a substance can take before breaking when stretched or pushed. The composites' tensile strength was determined using the Hounsfield Monsanto Tensometer 8889 and the American Standard Testing Method D-638-14. The dimensions of the test component were $160 \times 19 \times 3.2 \mathrm{~mm}$.

\subsubsection{Hardness Test}

The distance of indentation and recovery that happens when an indentor is pressed into the surface under constant load and then released is used to determine hardness. The surface hardness was estimated using the Hounsfield Monsanto Tensometer 8889 and the Brinell Hardness Number in $\mathrm{N} / \mathrm{mm}^{2}$ in line with the American Standard Testing Method E10. The test piece was $20 \times 20 \times 3.2 \mathrm{~mm}$ in size.

\subsubsection{Shear Strength}

A material's ability to withstand forces that cause its internal structure to slide against itself is known as shear strength.

The shear strength of the composites was determined using the Hounsfield Monsanto Tensometer 8889 and the American Standard Testing Method D-732. The dimensions of the test piece were $20 \times 20 \mathrm{~mm}$.

\subsubsection{Compressive Strength}

Compressive strength refers to a composite's ability to endure loads that cause it to shrink in size. It's just the ability to withstand compression.

The compressive strength of the composites was determined using the Hounsfield Monsanto Tensometer 8889 and the American Standard Testing Method D-695. The dimensions of the test piece were $40 \times 40 \mathrm{~mm}$.

\subsection{Water Absorption Test}

This analysis is to determine extent of water of the composites according to ASTMD-570-98. The composites of $50 \times 50 \mathrm{~mm}$ dimensions were dried before being immersed in water for three days. The increase in the weight of the material at daily intervals was used to determine how much moisture the composite absorbed. The percentage moisture absorption capacity is calculated as the ratio of the composite's increased mass to its original mass.

\subsection{Biodegradation Test}

This test is carried out to see how rapidly composites degrade in the environment. A soil burial degradation test was used to determine this. For degradation, LDPE composites were buried in soil taken from an automobile mechanic workshop. The mass reduction of composites buried was used to determine degradation.

Composites were cut into 50x50mm squares, weighed, and buried for three months at a depth of $15 \mathrm{~cm}$ in the soil. During the test period, the composites were weighed every 30 days to see the extent of degradation.

\subsection{Morphological Analysis}

To analyze the surface distribution between the polymer matrix and the filler, morphological examination was carried out with a Scanning Electron Microscope PRO:X:800-07334 Phenom world MVE01570775. 


\section{Result and Discussion}

\subsection{Mechanical Properties}

\subsubsection{Tensile Strength}

The impact of filler loading on the tensile strength of the composite is shown in Figure 1.

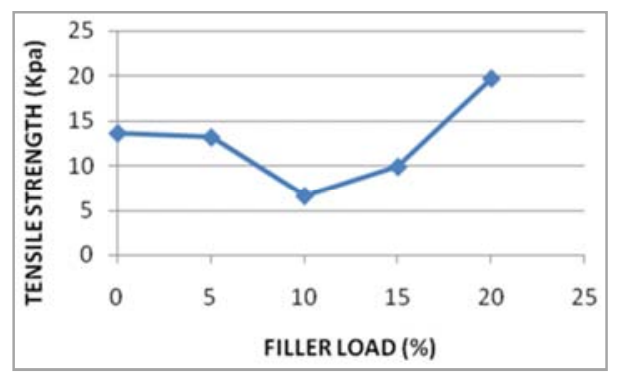

Figure 1. Impact of filler content on the tensile strength of LDPE composites

Figure 1 shows a reduction in the tensile strength of the composites as filler content increases, having the maximum value observed at $20 \mathrm{wt} \%$ filler load.

According to some researchers, the decrease in tensile strength is as a result of weak bonding between the fillers and the matrix and the cluster of filler particles in the matrix. The strength of the composite lies greatly on the bonding between the matrix and the filler. This will enable a small fragment of stress to be transferred to the filler particle during deformation. This decrease could also be attributed to an irregular arrangement of the fillers particle in the polymer matrix that may lead to more filler-filler interactions than the expected filler-matrix interaction.

The decrease observed in the value of the tensile strength of the composites is similar to other research studies on fillers obtained from renewable sources of energy, such as snail shell [12-16]. The $44 \%$ increase in the tensile strength at $20 \mathrm{wt} \%$ filler content is due to stronger bonding between filler and polymer matrix which leads to better stress transfer from the matrix to the filler [17].

\subsubsection{Percentage Elongation at Break}

This is the degree of ductility of the composite materials. Also known as fracture strain, it is the ratio of increased length and initial length measured after rupture of the test material. The impact of filler content on the percentage elongation at break of the composite is shown in Figure 2.

As seen in Figure 2, percentage elongation at break increased with an increase in filler content. This suggests that increasing filler loading in the polymer matrix caused the composites to become more ductile and malleable.

This increase is due to the addition of the natural fillers which causes an increase in the elasticity of the composite, and as a result, decreases the strength of the material [18].

A researcher [19] opined that the resulting increase of the elongation at break with the increasing filler content indicates the filler ability to enable the stress transfer from polymer filler to matrix.

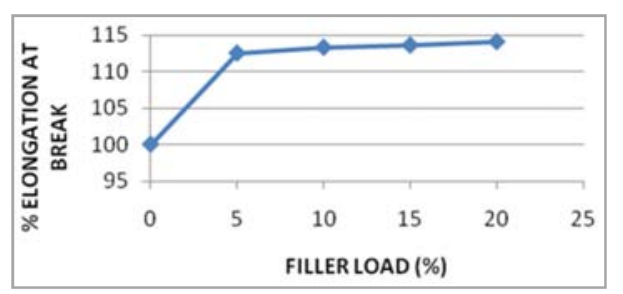

Figure 2. Impact of filler content on \% elongation at break.

\subsubsection{Hardness}

The hardness of the composites is its resistance to penetration, indentation, and deformation. The impact of filler loading on the hardness of the composite is shown in Figure 3.

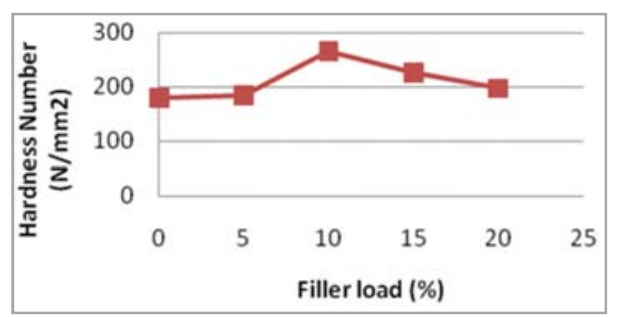

Figure 3. Impact of filler content on the hardness of LDPE composites.

The hardness value as observed from Figure 3 exhibited an overall increase as the filler content increases. This is an indication that the fillers enhanced the hardness of the composites with the composite exhibiting the highest hardness value at $10 \mathrm{wt} \%$ filler content.

The increase in hardness could be attributed to the filler's strengthening activity in the polymer matrix. Fillers are commonly used to improve the stiffness and strength of polymeric materials. This could possibly be due to the fact that adding fillers tightened the composites' elasticity and enhanced the matrix surface resistance to indentation [20].

\subsubsection{Compressive Strength}

This is the residual strain left in a test sample after it has been subjected to stress for a given time and allowed to recover on removing the deforming load. The impact of filler content on the compressive strength of the composite is shown in Figure 4.

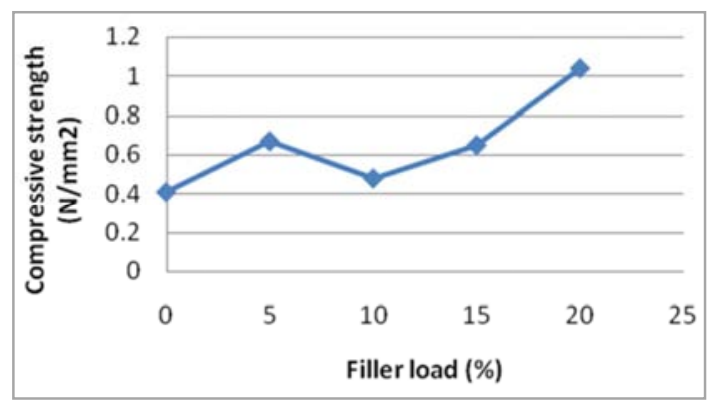

Figure 4. Impact of filler content on the compressive strength of LDPE composites.

Figure 4 shows that the composites' compressive strength has increased, with the optimum compressive strength at $20 \mathrm{wt} \%$ filler load increasing by $61 \%$. 
This increase could be attributable to the filler's reinforcing characteristics, which improve the composite's mechanical properties due to its cellulosic nature, and in agreement with the works of these researchers [21, 22].

\subsubsection{Shear Modulus}

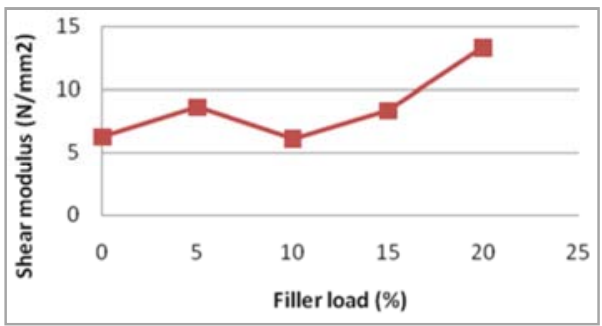

Figure 5. Impact of filler content on the Shear modulus of LDPE composites.

The shear modulus of a material is the modulus of rigidity of the material. It is calculated by measuring the deformation of a material by applying a force parallel to its surface, while an opposing force operates on the opposite surface and holds it in place. A fluid is defined as a substance having a zero shear modulus that deforms its surface when subjected to force. Figure 5 depicts the influence of filler loading on the composites' shear modulus.

As can be seen in Figure 5, the shear modulus increases as the filler loading increases. This increase suggests that the fillers increased the rigidity of the composites; a larger force is required to deform the composites along the plane of the direction of the force.

Ultimately, the harder a material/substance is the higher its shear modulus value.

\subsection{Morphological Analysis}

Scanning Electron Microscopy (SEM) is an effective media for the investigation of the surface morphology of the composites.

The dispersion and compatibility of the filler and the polymer matrix may be studied using SEM. The differences in the mechanical properties of the composites could be related to the SEM micrographs. During the analysis of the morphology of the composites, the following results were obtained. Figures 6(a), 6(b), 6(c), 6(d) are the micrograph of CCSB-LDPE 0wt $\%$, 5wt $\%, 10 w t \%$, and $15 \mathrm{wt} \%$, respectively.

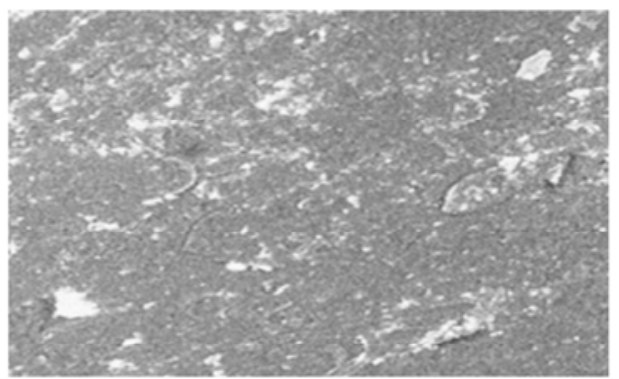

(a)

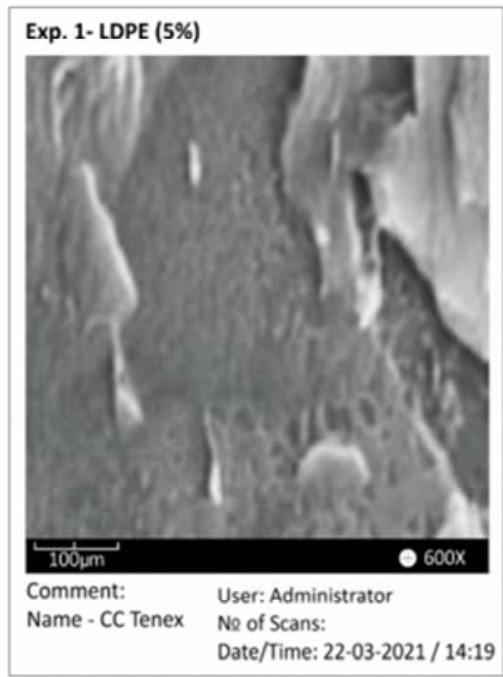

(b)

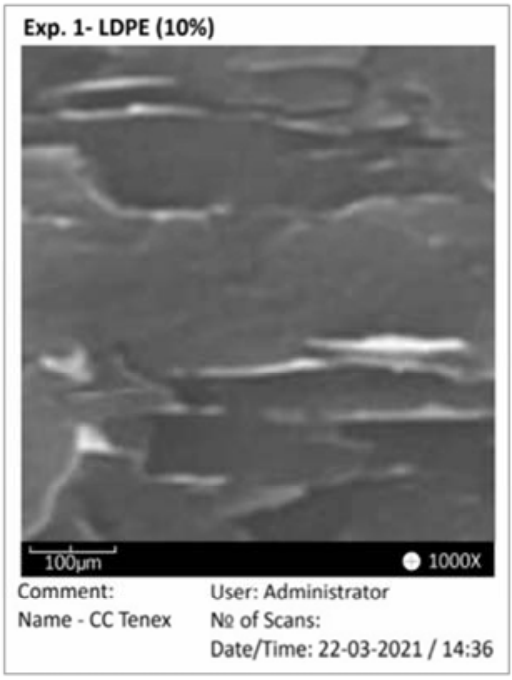

(c)

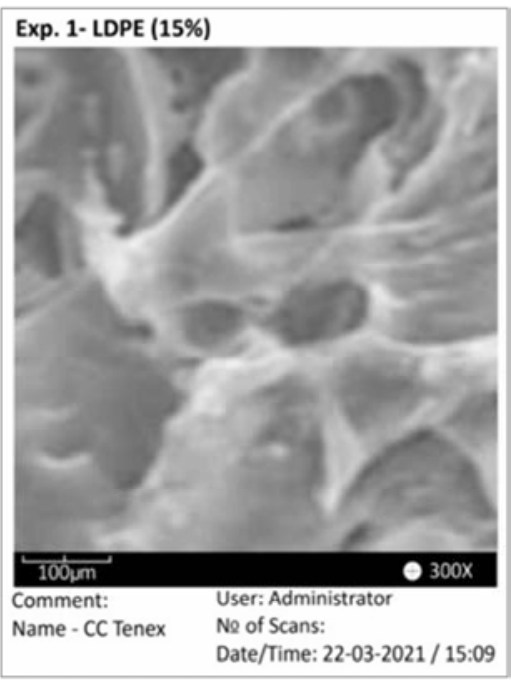

(d)

Figure 6. SEM micrographs of CCSB-LDPE composites.

Figure 6 shows that increase in the filler content improved the filler dispersion. The filler particles were properly bonded to the matrix, and it was clear that they were deeply embedded in the matrix. The great mixing efficiency was attributable to the high interfacial contact between the hybrid fillers and the polymer matrix. The composites' compatibility is improved by the fillers' uniform dispersion in the polymer matrix. This finding shows that the polymer matrix and filler have excellent adhesion. As a result, the interfacial strength has increased [23]. 


\subsection{Water Absorption}

The water imbibition results of the composites showed no increase to the final weight of the composites after complete immersion in water. This may be due to the hydrophobic nature of the polymer matrix and lignin-a component of the filler. Besides, the lower proportion of the filler content to the polymer matrix, and the degree of homogeneity in the dispersion of fillers may also be a factor. This result suggests that the composites can be used in wet environment; as water storage tanks, bathroom interior, etc.

\subsection{Biodegradation Test}

This was carried out to determine the extent the composites will degrade in the environment. For the purpose of this analysis, the least filled composites and the most filled composites were analyzed ( $5 \mathrm{wt} \%$ and $20 \mathrm{wt} \%$ respectively). Figure 7 reports the degradation test results for the produced LDPE composites.

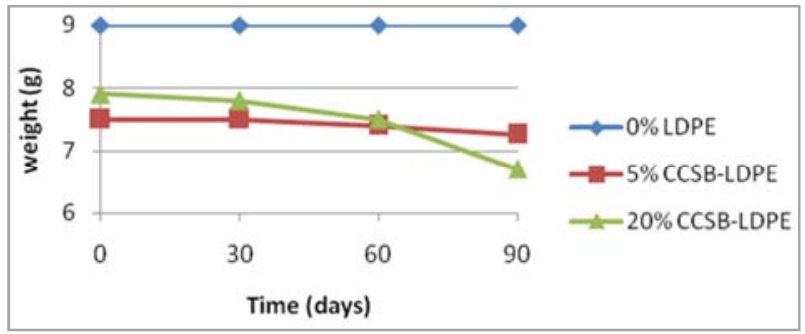

Figure 7. Impact of filler content on the degradation of composites.

From Figures 7, as anticipated, there was no reduction in weight for $0 \%$ LDPE during the 90 days test period. For $5 \mathrm{wt} \%$ composites, weight loss was noticed after 30 days during test period, and there was a general reduction in weight with as the burial time increased. It is also observed from the figure that increased filler content led to higher degradation of the composites. This observation corroborates the research findings of these authors [24, 25]. These authors discovered that biodegradability of composites is well pronounced and enhanced if the composite contains at least $30 \%$ of the natural fillers. The highest degradation rate was observed at $20 \mathrm{wt} \%$ filler loading giving a 15\% reduction after 90 days.

\section{Conclusion}

Corn cobs and sugarcane bagasse were successfully incorporated as hybrid fillers into low density polyethene (LDPE) polymer matrix to make polymer composites. The incorporation of this filler enhanced the percentage elongation at break, hardness, compressive strength, and shear modulus of the composite. However, the tensile strength decreased.

This finding is in agreement with existing literature and, shows that this is a common occurrence with lignocellulosic fillers. The synergy between the polymer matrix with the filler, as well as the particle size and dispersion of the fillers within the matrix, was discovered to influence the mechanical properties of the composites created.

The composites were found to resist water absorption over the testing period. This means the composite is ideal for water storage, drainage pipes, and other wet-environment applications.

The composites were also discovered to degrade, as evidenced by their weight loss during a three-month test period. More filler in the composites would considerably improve the composites' biodegradability. However, this may deteriorate the mechanical properties of the composites.

As a result of the findings, it is suggested that agro-wastes like rice husk and sugarcane bagasse be employed as fillers in the manufacture of plastics because they are cheaper and viable. Finally, the produced plastics would degrade when discarded.

\section{Recommendations}

1. Surface modifications are recommended employed to enhance the performance of lignocellulosic fillers and to promote better adhesion between the natural reinforcement and the polymeric matrix.

2. Further studies on the suitability of other readily available, eco-friendly and cheap natural materials as fillers in polymer composites.

\section{References}

[1] Ofora, P. U., Nwokoye, J. N., Ekpunobi, E. U., Okpalaunegbu, C. A., Ezeanyika, M., (2016). Effects of Bamboo-Corn Husk as Fillers on the Mechanical Properties in Polypropylene. International Journal of Materials Chemistry and Physics, 2 (2): 88-92.

[2] Chitra, J. N., and Vasanthakumari, R., (2012). Studies on polypropylene biocomposite with cornhusk waste. International Journal of Scientific and Engineering Research, 3 (7): 562-564.

[3] Vidyashri, V., Lewis, H., Narayanasamy, P., Mahesha, G. T., Bhat, K. S., (2019). Preparation of chemically treated sugarcane bagasse fiber reinforced epoxy composites and their characterization. Cogent Engineering, 6 (1): 1-20.

[4] Agunsoye, J. O., and Aigbodion, V. S. (2013)., Bagasse filled recycled polyethene bio-composites: Morphological and mechanical properties study. Results in Physics, 3: 187-194.

[5] Thakur, V. K., and Kessler, M. R., (2014). Free radical induced graft copolymerization of ethyl acrylate onto soy for multifunctional materials. Materials Today Communications, 1 (1-2): 34-41.

[6] Chen, R. S., Ab Ghani, M. H., Ahmad, S., Salleh, M. N., Tarawneh, M. A. A., (2015). Rice husk flour biocomposites based on recycled high-density polyethene/polyethene terephthalate blend: Effect of high filler loading on physical, mechanical and thermal properties. Journal of Composite Materials, 49 (10): 1241-1253.

[7] Kumar, S., Mer, K. K. S., Gangil, B., Patel, V. K., (2019). Synergy of rice-husk filler on physico-mechanical and tribological properties of hybrid Bauhinia-vahlii/sisal fiber reinforced epoxy composites. Journal of Materials Research and Technology, 8 (2): 2070-2082. 
[8] Mishra, A., (2017). Mechanical properties of cocconut shell dust, epoxy-fly ash hybrid composites. American Journal of Engineering Research, 6 (9): 166-174.

[9] Bekele, L. D., Zhang, W., Liu, Y., Duns, G. J., Yu, C., Jin, L., Li, X., Chen, J., (2017). Impact of cotton stalk biomass weathering on the mechanical and thermal properties of cotton stalk flour/linear low density polyethene (LLDPE) composites. Journal of Biobased Materials and Bioenergy, 11 (1): 27-33.

[10] Nguyen, H., Moghadam, M. J., Moayedi, H., (2019). Agricultural wastes preparation, management, and applications in civil engineering: a review. Journal of Material Cycles and Waste Management, 21: 1039-1051.

[11] Prithivirajan, R., Jayabal, S., Bharathiraja, G., (2015). Biobased composites from waste agricultural residues: Mechanical and morphological properties. Cellulose Chemistry and Technology, 49 (1): 65-68.

[12] Salmah, H., Ismail, A., Bakar A., (2005). Dynamic vulcanization of paper sludge filled polypropylene (PP)/ethylene propylene dienetherpolymer (EPDM). Malaysian Journal of Microscopy, 2: 15-22.

[13] Chris-Okafor P. U, Arinze R. U, Ekpunobi U. E, Anugwom M. C., (2018). Effects of mixed rice husk and corn cob as fillers on some properties of flexible polyether foam. Global Journal of Science Frontier Research, 17 (2): 30-37.

[14] Zhang, Q., Weiming, Y., Zhihe, Li., Lihong, W., and Hongzhen C., (2018). Mechanical Properties of Rice Husk Biochar Reinforced High Density Polyethene Composites, Polymers, 10: 286-296.

[15] Maya Jacob; Sabu Thomas; K. T. Varughese (2004). Mechanical properties of sisal/oil palm hybrid fiber reinforced natural rubber composites. Composites Science and Technology 64 (7-8): 955-965.

[16] Cautinho, f. M. B., Caota, T. H. S. and Carvalho, d. L. (1997). Polypropylene-wood fiber composites: Effect of treatment and mixing condition on mechanical properties. Composites, 43: 154-159.
[17] Morreale, M., Scaffaro, R., Maio, A. \& La Mantia, F. P. (2007) Effect of adding wood flour to the physical properties of a biodegradable polymer. Composite, 42: 214-225.

[18] Abdullah, A., Rusel, D., and Abdulwahab, A., (2011). Water absorption and mechanical properties of high - density polyethene/ egg shell composite. Journal of Basrah Researches (Sciences), 37 (15): 1-8.

[19] Kricheldorf, H., Nuyken, O., and Swift, G., (2005). Hand book of Polymer Synthesis, Second Edition, Marcel Dekker.

[20] Chris-Okafor, P., Okonkwo, C., Ohaeke, M., (2018). Reinforcement of High Density Polyethene with Snail Shell Powder, American Journal of Polymer Science, 8 (1): 17-21.

[21] Onuegbu T. U., (2012); Study on the Effect of Coconut and Palm Kernel Shells on Density, Porosity Index and Tensile Properties of Flexible Polyether Foam. ARPN Journal of Science and Technology 2 (6).

[22] Kuan, C. f., Kuan, H. C., Ma, C. C. M. \& Haung, c. M. (2006) Mechanical, thermal and morphological properties of watercrosslinked wood flour reinforced linear low-density polyethene composites, Composites, Part A, 37: 1696-1707.

[23] Amir, N., Ashori, A., Kazemi T., Ali, (2014). Characterization and biodegradability of polypropylene composites using agricultural residues and waste fish. Composites Part B: Engineering, 56 (2014): 279-283.

[24] Oldak, D., Kaczmarek, H., Buffeteau, T., Sourisseau, C., (2006). Photo and bio-degradation processes in polyethene, cellulose and their blends studied by ATR-FTIR and Raman spectroscopies. Journal of Material Science; 40 (16): 4189 4198.

[25] Nwanonenyi, S. C., Obidiegwu, M. U., Onuegbu, G. C., (2013). Effects of Particle Sizes, Filler Contents and Compatibilization on the Properties of Linear Low Density Polyethene Filled Periwinkle Shell Powder. The International Journal of Engineering and Science (IJES), 2 (2): 1-8. 